\title{
Posterior Positioning of a Clavicle Hook Plate Is a Risk Factor for Acromial Fractures after Acromioclavicular Joint Dislocation
}

\section{Hironari Tamiya1,2, Shusaku Umemoto1, Yasuyoshi Akimoto', Takayuki Kyo', Kazushige Gamo', Shigeyuki Kuratsu ${ }^{1}$}

${ }^{1}$ Department of Orthopedic Surgery, Bellland General Hospital, Osaka, Japan

${ }^{2}$ Department of Orthopedic Surgery, Osaka International Cancer Institute, Osaka, Japan

Email: tamiya-hi@mc.pref.osaka.jp

How to cite this paper: Tamiya, $\mathrm{H}$., Umemoto, S., Akimoto, Y., Kyo, T., Gamo, K. and Kuratsu, S. (2019) Posterior Positioning of a Clavicle Hook Plate Is a Risk Factor for Acromial Fractures after Acromioclavicular Joint Dislocation. Open Journal of Orthopedics, 9, 101-111. https://doi.org/10.4236/ojo.2019.94010

Received: March 17, 2019

Accepted: April 15, 2019

Published: April 18, 2019

Copyright $\odot 2019$ by author(s) and Scientific Research Publishing Inc. This work is licensed under the Creative Commons Attribution International License (CC BY 4.0).

http://creativecommons.org/licenses/by/4.0/

\begin{abstract}
Purpose: Acromioclavicular (AC) joint dislocation is commonly treated using a clavicle hook plate (HP). However, previous reports have indicated that acromial fractures may occur after HP fixation. The purpose of this study was to identify risk factors for acromial fractures. Methods: A retrospective study was conducted on 39 patients with AC joint dislocation who were treated using clavicle HP fixation in our hospital between 2006 and 2017. Related parameters, including Rockwood classification, hook angle, the degree of reduction, the coverage of the hook under the acromion, and the anteroposterior position of the hook under the acromion, were evaluated to identify risk factors for acromial fractures. Results: The mean age of the participants was 51.7 (range 19 - 81) years; 34 were men and 5 were women. Injury occurred on the right side in 18 patients and on the left side in 21 . Injuries were categorized as follows: 24 were Rockwood type III, one was type IV, and 14 were type V. Four of the 39 patients (10\%) experienced acromial fractures. Statistical analyses indicated that the degree of reduction at the final follow-up was moderately correlated with the Constant score. Posterior positioning of the hook was the only identified risk factor for acromial fractures. Hook angle and the degree of reduction at the time of surgery were not significantly associated with acromial fractures. Conclusions: Postoperative shoulder function was associated with the degree of reduction at the final follow-up, suggesting that anatomical reduction is recommended for AC joint dislocation. Posterior positioning of the hook is a risk factor for acromial fractures; however, clavicle HP fixation provides a positive outcome for AC joint dislocation. Therefore, careful positioning of the hook is required for preventing acromial fractures.
\end{abstract}




\section{Keywords}

Acromioclavicular Joint Dislocation, Clavicle Hook Plate, Subacromial Osteolysis, Acromial Fracture

\section{Introduction}

Acromioclavicular (AC) joint dislocation is a frequently occurring injury, particularly among young and middle-aged people. In Rockwood type I and II AC injuries, the joint is intact or minimally displaced, respectively, and can be nonsurgically treated. On the contrary, surgical treatment can provide better outcomes for Rockwood type III - VI AC injuries than conservative therapy [1]. Among the fixation methods, clavicle hook plate (HP) fixation has been widely used. However, previous reports have indicated that HP fixation causes certain complications, including subacromial impingement, proximal clavicular fractures, subacromial bony erosion, and acromial fractures. In particular, subacromial erosion and acromial fractures occasionally occur because the hook placed under the acromion erodes, loading an excessive stress on the acromion. Rotational movement of the clavicle during shoulder joint motion is one of the possible causes of subacromial erosion and acromial fractures [2]; however, the precise underlying mechanisms are yet to be determined. In the present study, we retrospectively studied 39 patients with AC joint dislocation, wherein the dislocations were repaired using HP fixation; among these, four patients (10\%) experienced acromial fractures. We investigated a selection of parameters, including Rockwood classification, hook angle, the degree of reduction, and the coverage of the hook under the acromion to identify risk factors for acromial fractures.

\section{Material and Methods}

All patients were treated in Bellland General Hospital between 2006 and 2017. All patients treated with HP fixation against AC joint dislocation were enrolled in the present study. Exclusion criteria were revision from other operative methods. HPs from two different manufacturers (DePuy Synthes, Warsaw, IN, USA and Homs, Nagano, Japan) were used. The plates were selected according to the preference of the clinician performing the surgery. The surgical technique was performed as follows: patients underwent surgery in beach chair position under general anesthesia with the affected arm in a freely movable state. A transverse incision was made along the clavicle over the AC joint. The AC joint dislocation was identified; then the clavicle HP was placed to reduce the dislocation. Repair of the ruptured AC ligament was performed according to the preference of the clinician. The degree of subacromial osteolysis was evaluated using standard $\mathrm{x}$-ray and scored as follows: $0=$ no osteolysis, $1=$ partial osteolysis without perforation, 2 = partial perforation, and 3 = acromial fracture. Representative $\mathrm{x}$-rays are shown in Figures 1(a)-(d). 


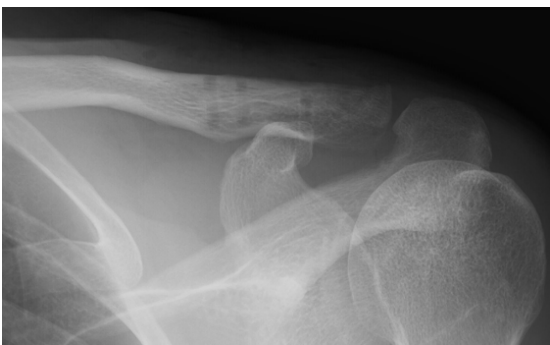

(a)

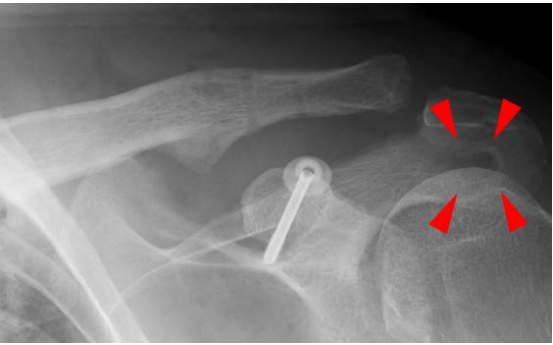

(b)

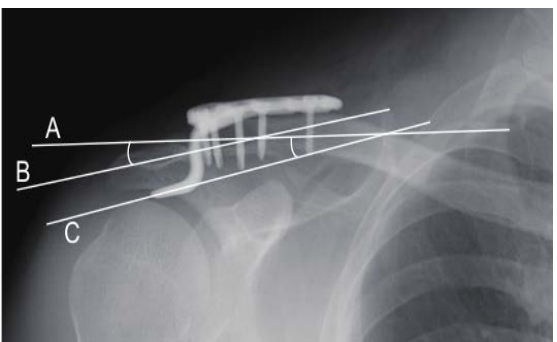

(e)

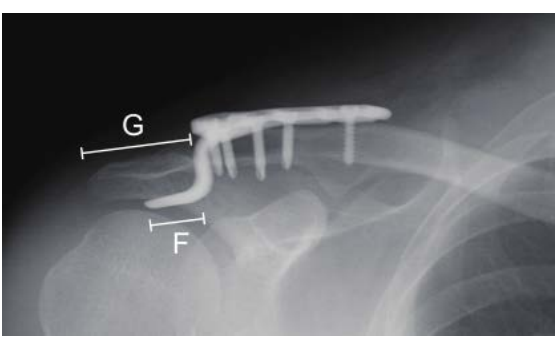

(g)

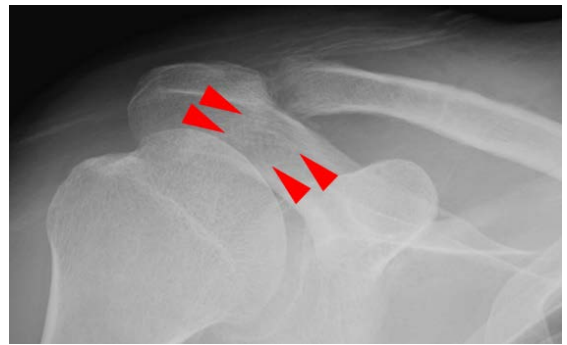

(c)

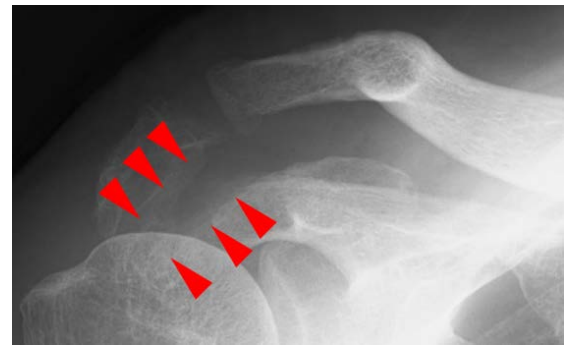

(d)

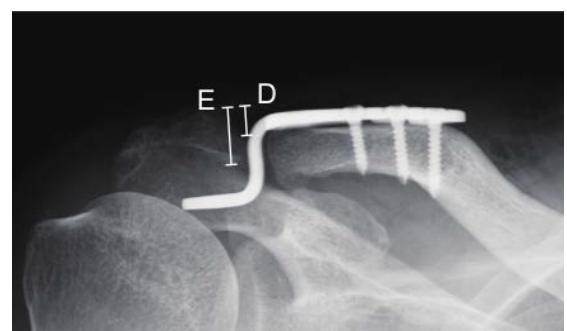

(f)

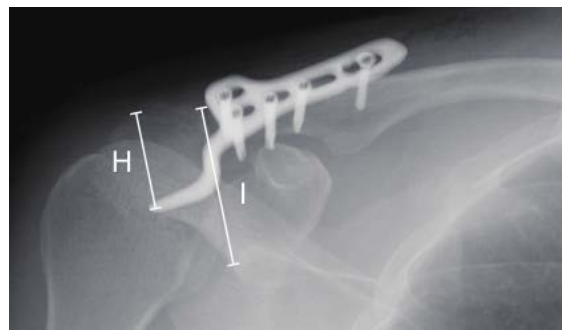

(h)

Figure 1. Representative plain radiograph showing subacromial osteolysis with definitions of each parameter evaluated in this study. Representative plain radiographs of osteolysis graded as 0 (a), 1 (b), 2 (c), and 3 (d) are shown. Red arrowheads highlight the areas of osteolysis. Plain radiographs illustrate the parameters as defined in this study (e)-(h).

The parameters used in this study are defined in Figures $1(\mathrm{e})-(\mathrm{h})$. The AC angle was defined as the degree of angle produced by lines A and B, where line A represented the axis of the distal third of the clavicle and line $B$ represented the axis of the acromion. The hook angle was determined by crossing lines B and C, where line $\mathrm{C}$ was the axis of the hook. The degree of reduction was calculated by the ratio of $\mathrm{D}$ to $\mathrm{E}$, where $\mathrm{D}$ was the distance between the proximal line of the 
clavicle and that of the acromion and $\mathrm{E}$ was the thickness of the acromion; a positive value represented over-reduction. Coverage of the hook was defined as the ratio of $\mathrm{F}$ to $\mathrm{G}$, where $\mathrm{F}$ was the distance of the hook placed under the acromion and $G$ was the transverse length of the acromion. The anteroposterior (A-P) position was measured by the ratio of $\mathrm{H}$ to $\mathrm{I}$, where $\mathrm{H}$ was the distance between the posterior edge of the acromion and tip of the hook and I was the entire length from the posterior to anterior edge parallel to line $\mathrm{H}$.

As an indicator of shoulder function, the Constant score was adopted as previously described [3]. The scoring system comprised of the following four categories: pain (15 points), activities of daily living (20 points), range of motion (40 points), and strength ( 25 points). Pain was assessed according to the degree of pain (0 - 15 points). Activities of daily living comprised sleep ( $0-2$ points), recreation/sport ( 0 - 4 points), work ( 0 - 4 points), and arm positioning (0 - 10 points). Range of motion was evaluated by the degree of elevation $(0-10$ points), abduction ( 0 - 10 points), external rotation ( $0-10$ points), and internal rotation ( 0 - 10 points). Strength of abduction was measured ( 0 - 5 points). Pain was evaluated according to visual analog scale (VAS). VAS score 1 - 3 was regarded as mild, 4 - 6 moderate, and 7 - 10 was defined as severe pain.

Student's t-test and Pearson's correlation were used for statistical analyses of differences and relationships between two parameters, respectively. The p-value for Pearson's correlation was calculated using Microsoft Excel 2016 using the following equation: $\mathrm{p}=\operatorname{TDIST}\left(\mathrm{r}^{*} \operatorname{SQRT}(\mathrm{n}-2) / \operatorname{SQRT}\left(1-\mathrm{r}^{2}\right), \mathrm{n}-2,2\right)$. For analyzing trends among multiple groups, the Jonckheere-Terpstra test was adopted using EZR [4]. For comparisons between multiple groups, Dunnett's test was adopted [5]. A p-value of less than 0.05 was considered statistically significant.

\section{Results}

All parameters analyzed in this study are shown in Table 1. In total, 34 men and five women were included; their mean age was 51.7 years (range 19 - 81 years). Overall, 18 patients had injured their right shoulder and 21 had injured their left. Twenty-four patients were classified as having Rockwood type III AC injury, one was classified as type IV, and 14 were classified as type V. Among the 39 patients, 14 (36\%) exhibited no osteolysis, 16 (41\%) showed minor subacromial osteolysis, five (13\%) presented major osteolysis (partial perforation of the acromion), and four (10\%) had acromial fractures.

We analyzed the correlations between parameters and found that the degree of reduction at the final follow-up and the Constant score were moderately correlated $(r=0.438, p=0.005$; Figure 2). Furthermore, the degree of reduction at the final follow-up and osteolysis grade showed a significant trend toward being correlated $(\mathrm{p}=0.008)$. These results imply that the degree of reduction is associated with clinical function and reduction is lost with subacromial osteolysis. Over-reduction at the time of surgery and osteolysis grade $(\mathrm{p}=0.727)$, difference of AC hook angles and osteolysis grade $(\mathrm{p}=0.446)$, and coverage of the hook 
产焉|

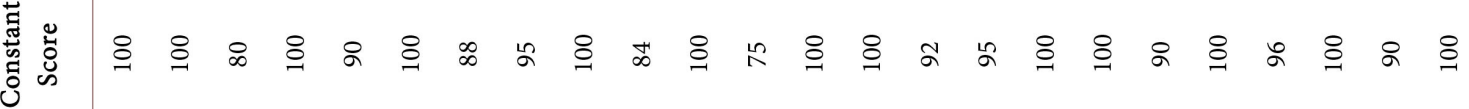

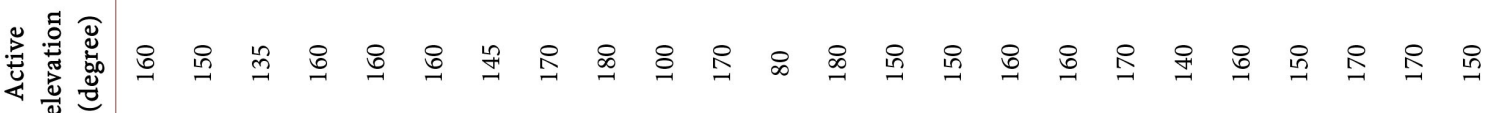

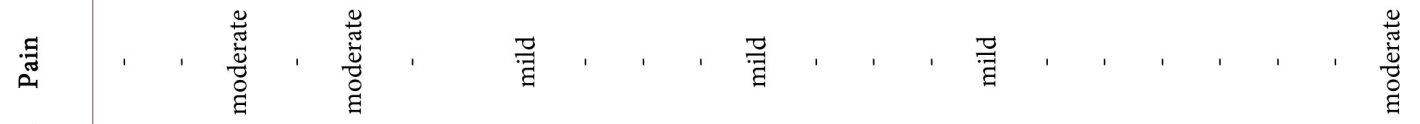

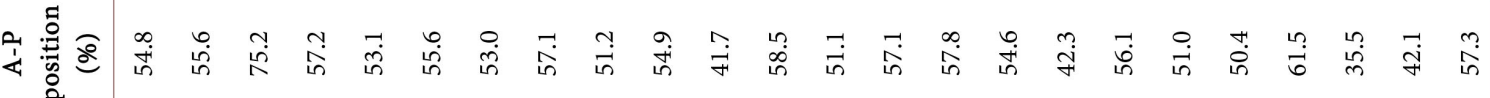

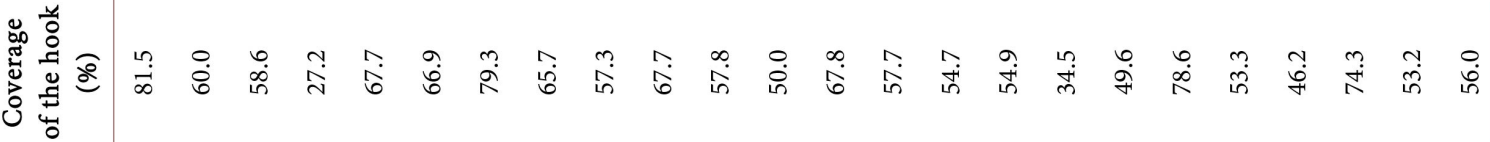

苞苟

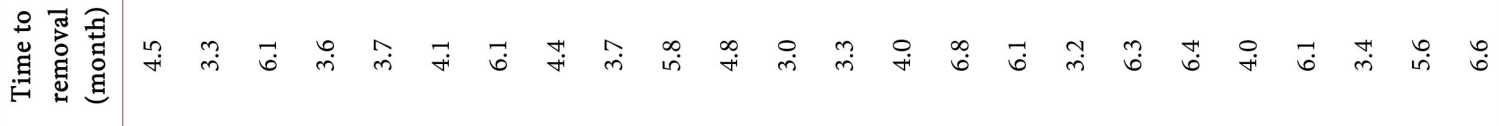

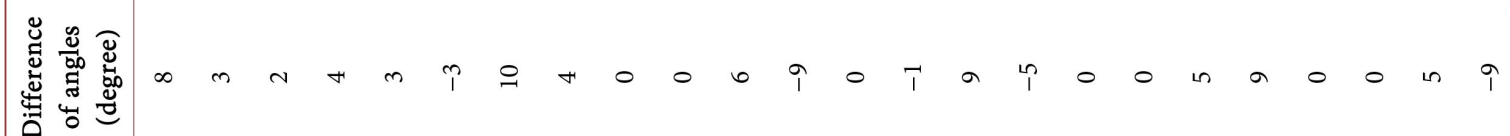

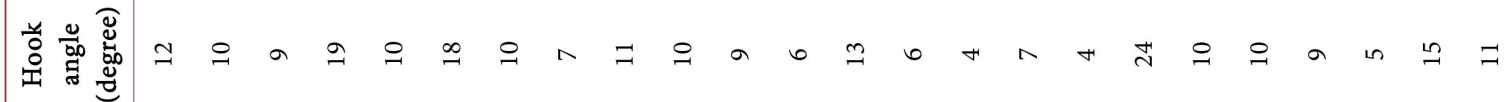

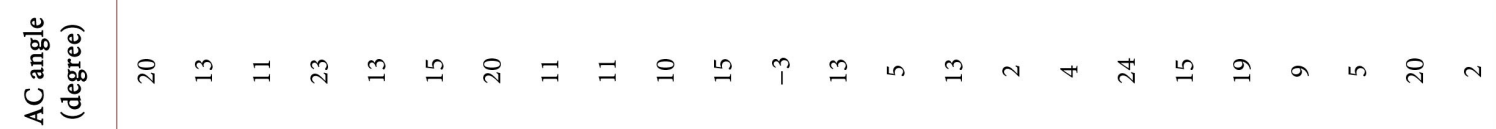
焉

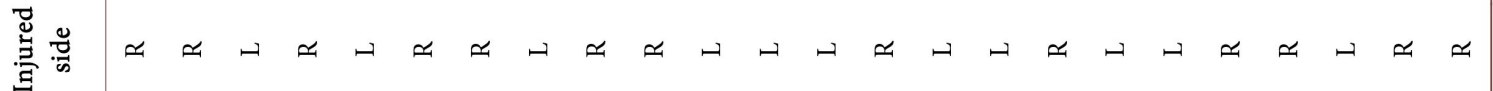
桇

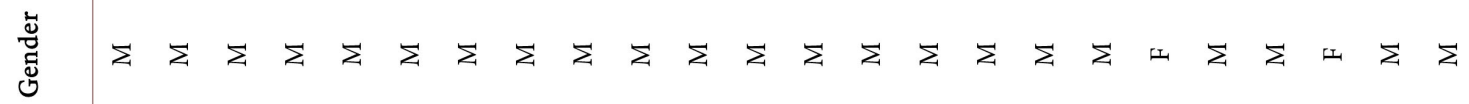

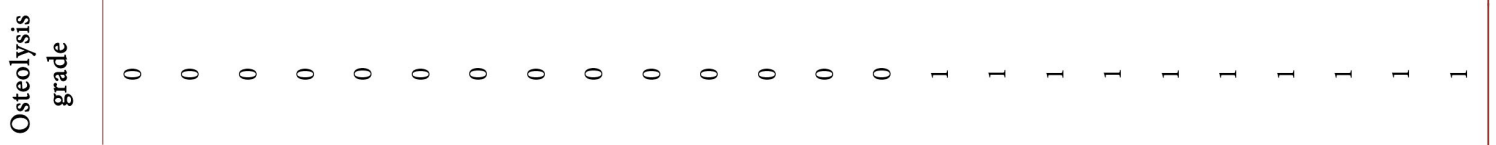




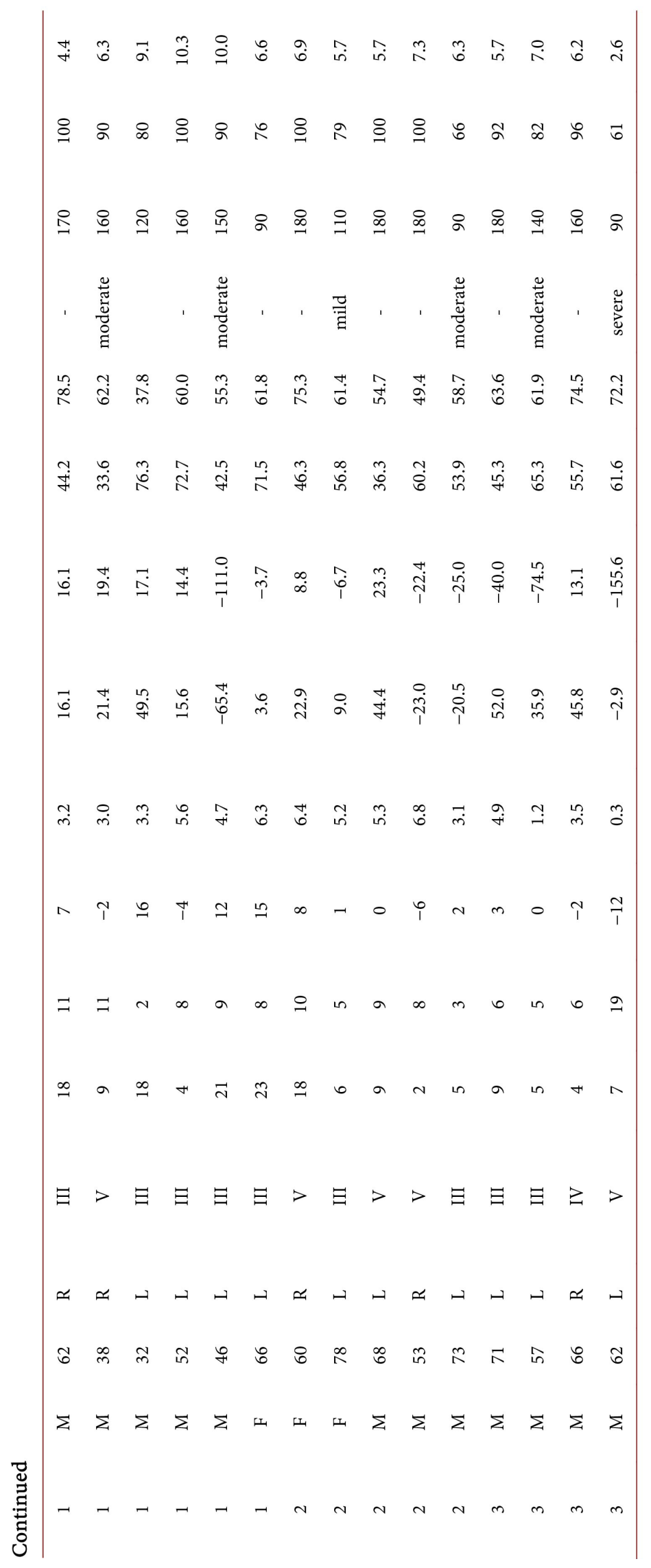




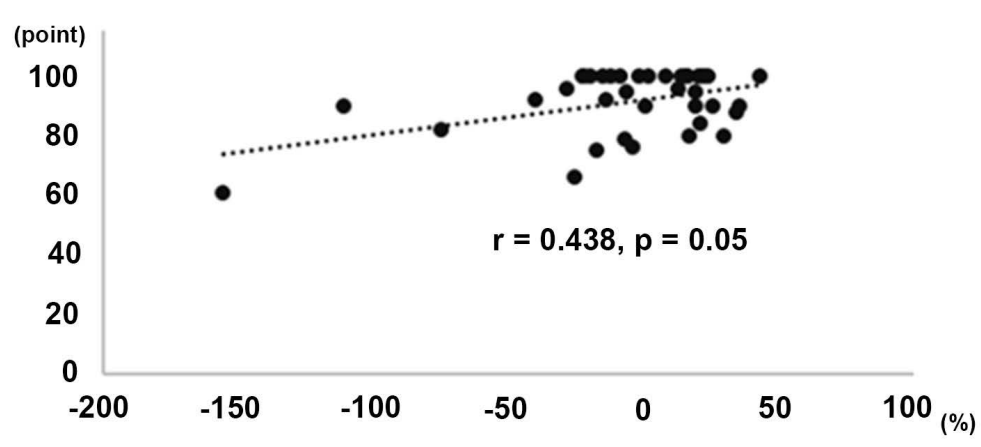

Figure 2. Correlation between the degree of reduction at the final follow-up and the Constant score. The dotted line represents the approximate line of best fit.

and osteolysis grade $(\mathrm{p}=0.101)$ were not significantly correlated. Notably, A-P position of the hook and osteolysis grade were significantly correlated ( $\mathrm{p}=$ 0.039). In addition, a comparison between osteolysis grades 1 and 3 demonstrated a significant difference in the associated A-P position $(\mathrm{p}=0.026)$ (Figure 3 ). Therefore, we concluded that posterior positioning of the hook was the only risk factor for subacromial osteolysis or acromial fractures among the investigated parameters.

\section{Discussion}

AC joint dislocation is frequently observed in young and middle-aged physically active individuals, and it occurs as a result of sports trauma or an accident. AC dislocation is divided into six types according to Rockwood classification, which provides an indication often used for determining the need for surgical treatment. Rockwood type I and II AC injuries can be nonsurgically treated. Treatment for type III AC injury is controversial, but surgical fixation has been recommended, particularly in active patients [1]. Surgical repair is recommended for type IV, V, and VI AC injuries. AC joint dislocation has been treated by several methods, such as tension band wiring or coracoclavicular ligament reconstruction, each with its own advantages and disadvantages. HPs provide a positive outcome; however, complications including subacromial osteolysis, acromial fractures, and subacromial impingement have been documented [6]. Another option is coracoclavicular reconstruction with the tightrope plus endobutton technique, which provides better function and lower postsurgical pain compared with HP fixation; however, higher complication rates, such as early subluxation and intrasurgical fracture have been reported with this approach [7] [8]. Presently, whether the tightrope method overcomes the requirement of HPs remains unclear, and further investigation is required to determine this.

The risk factors for acromial fractures have not yet been clarified. Specifically, two cases of acromial fractures after HP fixation were reported by Kang et al. [9]. They described the cause of those fractures as excessive shoulder motion and suggested that the HP should not be used in patients who are unable to follow a 


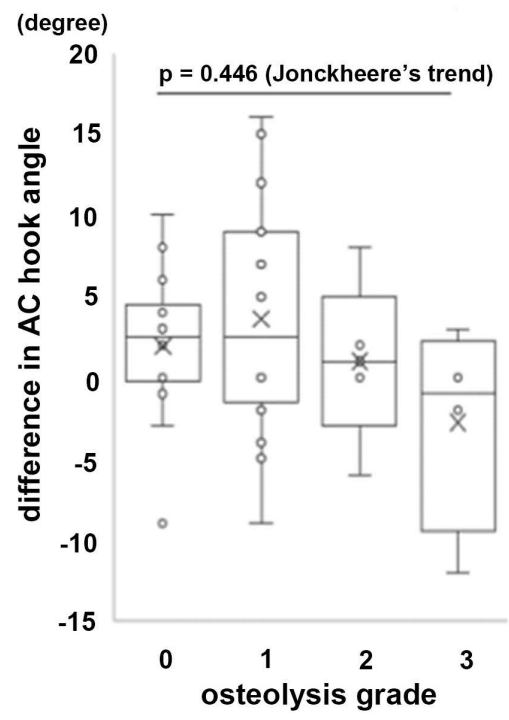

(a)

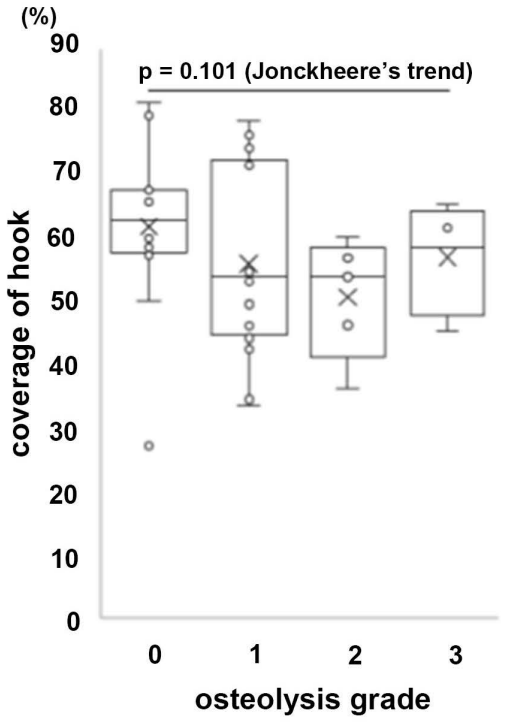

(c)

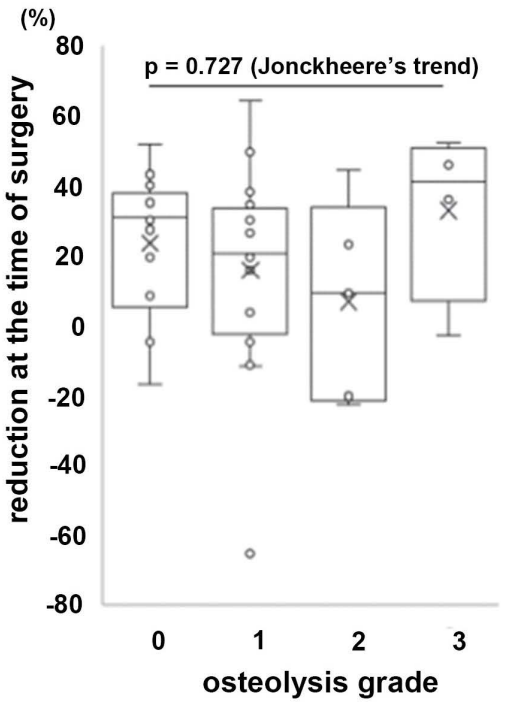

(b)

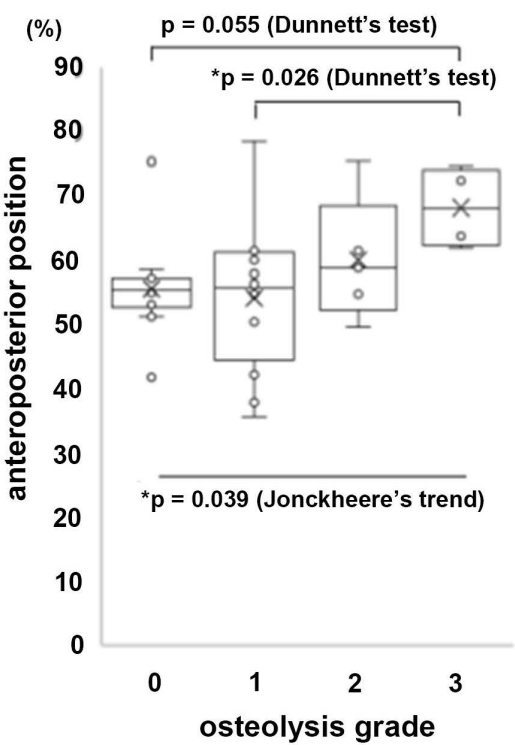

(d)

Figure 3. Comparisons between the degree of subacromial osteolysis and the individual factors evaluated in this study. Osteolysis grade was compared to the difference in AC hook angle (a), the degree of reduction at the time of surgery (b), coverage of the hook (c), and anteroposterior position (d). The asterisk ${ }^{*}$ ) indicates statistical significance.

routine rehabilitation program before plate removal. There are other reports that advise patients to restrict shoulder motion and avoid high intensity activities before implant removal [2] [10]. In the present study, one in four patients had a psychological condition that made adherence to prescribed restrictions challenging.

Another discussion point is the angle of the hook on the plate. Virtual implantation analysis for measuring the loading force on the acromion and clavicle has been conducted. Hung et al. [11]. Demonstrated that a larger hook angle produces more force on the acromion. On the contrary, a smaller hook increases the stress on the clavicle. The authors emphasized the importance of selecting 
the optimal angle for the HP for each patient. Another report also demonstrated that the HP makes a pinpoint contact between the hook and acromion, resulting in subacromial erosion [12]. For avoiding such complications, an HP that is tailored for the individual patient is required, because the AC angle largely varies between patients [13]. HPs with an angle greater than $90^{\circ}$ have been developed by several companies, which may reduce complications associated with subacromial erosion and/or cutout of the acromion [14]. Muramatsu et al. suggested that the plate be bent along the contour of the clavicle [15]. In the present study, the difference between the hook and AC angles was not significantly related to subacromial osteolysis. Furthermore, we investigated the extent to which the hook covered the acromion, as less coverage may increase the stress on the acromion, thereby triggering osteolysis of the acromion. However, our results demonstrated that there was no significant difference between the coverage of the hook and subacromial osteolysis.

With regard to the Constant score, there was no correlation between subacromial osteolysis and poor outcome when using the HP fixation for acute displaced lateral clavicle fractures [16]. Similarly, our study indicated that subacromial osteolysis was not significantly related to clinical outcome. On the contrary, our results suggested that postsurgical shoulder function was associated with the degree of reduction at the final follow-up. This means that anatomical reduction is beneficial in AC joint dislocation.

Lastly, positioning of the hook under the acromion should be considered. As previously studied, bone thickness varies depending on the portion of the acromion. The median thickness of the antero-medial and postero-medial portion of the acromion is 10.1 and $8.2 \mathrm{~mm}$, respectively, suggesting that the anterior portion tends to be thicker [17]. Additionally, the median angle of the acromial arch (on the sagittal plane) is $21.4^{\circ}$ (in females) and $23.3^{\circ}$ (in males), indicating that the osseous structure of acromion tilts downwards from the anterior to posterior side. This suggests that as the placement of the hook becomes more posterior, the occurrence of over-reduction may become more severe. Over-reduction is the one of the most plausible reasons of acromial cutout because of the stress it places on the acromion; however, this study demonstrated that over-reduction was not significantly associated with osteolysis.

There are some limitations in the present study. One is sample size. AC joint dislocation was less common than distal clavicular fractures; therefore only thirty-nine patients could be enrolled. To obtain more established conclusion, further study included larger number of the patients will be needed. Second one is retrospective study, which is generally less convincing than prospective study. The last one is our selection of operative method. The used plate and repair of AC ligament depended on each operator. This may mislead the conclusion.

\section{Conclusion}

In conclusion, the findings of our study suggest that the hook should be placed 
on the anterior side of the acromion for avoiding acromial fractures. It is crucial that subacromial impingement is considered; therefore, indications of impingement should be intrasurgically checked.

\section{Acknowledgements}

We would like to thank Enago (www.enago.jp) for the English language review.

\section{Conflicts of Interest}

The authors declare that they have no conflict of interest.

\section{References}

[1] Simovitch, R., Sanders, B., Ozbaydar, M., Lavery, K. and Warner, J.J.P. (2009) Acromioclavicular Joint Injuries: Diagnosis and Management. The Journal of the American Academy of Orthopaedic Surgeons, 17, 207-219. https://doi.org/10.5435/00124635-200904000-00002

[2] Charity, R., Haidar, S., Ghosh, S. and Tillu, A. (2006) Fixation Failure of the Clavicular Hook Plate: A Report of Three Cases. Journal of Orthopaedic Surgery, 14, 333-335. https://doi.org/10.1177/230949900601400320

[3] Constant, C.R., Gerber, C., Emery, R.J.H., Søjbjerg, J.O., Gohlke, F. and Boileau, P. (2008) A Review of the Constant Score: Modifications and Guidelines for Its Use. Journal of Shoulder and Elbow Surgery, 17, 355-361. https://doi.org/10.1016/j.jse.2007.06.022

[4] Kanda, Y. (2013) Investigation of the Freely Available Easy-to-Use Software "EZR" for Medical Statistics. Bone Marrow Transplantation, 48, 452-458.

https://doi.org/10.1038/bmt.2012.244

[5] Mikami, S., Hamano, T., Fujii, N. and Nagasawa, Y. (2008) Serum Osteoprotegerin as a Screening Tool for Coronary Artery Calcification Score in Diabetic Pre-Dialysis Patients. Hypertension Research, 31, 1163-1170.

https://doi.org/10.1291/hypres.31.1163

[6] Kashii, M., Inui, H. and Yamamoto, K. (2006) Surgical Treatment of Distal Clavicle Fractures Using the Clavicular Hook Plate. Clinical Orthopaedics and Related Research, 447, 158-164. https://doi.org/10.1097/01.blo.0000203469.66055.6a

[7] Arirachakaran, A., Boonard, M., Piyapittayanun, P., Kanchanatawan, W., Chaijenkij, K., Prommahachai, A. and Kongtharvonskul, J. (2017) Post-Operative Outcomes and Complications of Suspensory Loop Fixation Device versus Hook Plate in Acute Unstable Acromioclavicular Joint Dislocation: A Systematic Review and $\mathrm{Me}-$ ta-Analysis. Journal of Orthopaedics and Traumatology, 18, 293-304.

https://doi.org/10.1007/s10195-017-0451-1

[8] Zhang, L.F., Yin, B., Hou, S., Han, B. and Huang, D. (2017) Arthroscopic Fixation of Acute Acromioclavicular Joint Disruption with TightRope ${ }^{\mathrm{TM}}$ : Outcome and Complications after Minimum 2 (2-5) Years Follow-Up. Journal of Orthopaedic Surgery, 25, 2309499016684493. https://doi.org/10.1177/2309499016684493

[9] Kang, S., Lee, H.M. and Back, I.H. (2016) Acromion Fracture after Hook Plate Fixation for Distal Clavicle Injury: A Report of 2 Cases. Clinics in Shoulder and Elbow, 19, 168-171. https://doi.org/10.5397/cise.2016.19.3.168

[10] Hackenbruch, W., Regazzoni, P. and Schwyzer, K. (1994) Surgical Treatment of Lateral Clavicular Fracture with the "Clavicular Hooked Plate". Zeitschrift für Un- 
fallchirurgie und Versicherungsmedizin, 87, 145-152.

[11] Hung, L.K., Su, K., Lu, W.H. and Lee, C.H. (2017) Biomechanical Analysis of Clavicle Hook Plate Implantation with Different Hook Angles in the Acromioclavicular Joint. International Orthopaedics, 41, 1663-1669.

https://doi.org/10.1007/s00264-016-3384-z

[12] Yoon, J.P., Lee, Y.S., Song, G.S. and Oh, J.H. (2017) Morphological Analysis of Acromion and Hook Plate for the Fixation of Acromioclavicular Joint Dislocation. Knee Surgery, Sports Traumatology, Arthroscopy, 25, 980-986. https://doi.org/10.1007/s00167-016-3987-3

[13] DePalma, A.F. (1963) Surgical Anatomy of Acromioclavicular and Sternoclavicular Joints. The Surgical Clinics of North America, 43, 1541-1550.

https://doi.org/10.1016/S0039-6109(16)37142-0

[14] Cirstoiu, C., Radulescu, R., Popescu, D., Ene, R., Circota, G. and Badiceanu, C. (2009) Acroplate-A Modern Solution for the Treatment of Acromioclavicular Joint Dislocation. Journal of Medicine and Life, 2, 173-175.

[15] Muramatsu, K., Shigetomi, M., Matsunaga, T., Murata, Y. and Taguchi, T. (2007) Use of the AO Hook-Plate for Treatment of Unstable Fractures of the Distal Clavicle. Archives of Orthopaedic and Trauma Surgery, 127, 191-194.

https://doi.org/10.1007/s00402-006-0284-5

[16] Tiren, D., Van Bemmel, A.J.M., Swank, D.J. and Van Der Linden, F.M. (2012) Hook Plate Fixation of Acute Displaced Lateral Clavicle Fractures: Mid-Term Results and a Brief Literature Overview. Journal of Orthopaedic Surgery and Research, 7, 2. https://doi.org/10.1186/1749-799X-7-2

[17] Voss, A., Dyrna, F., Achtnich, A., Hoberman, A., Obopilwe, E., Imhoff, A.B., Mazzocca, A.D. and Beitzel, K. (2017) Acromion Morphology and Bone Mineral Density Distribution Suggest Favorable Fixation Points for Anatomic Acromioclavicular Reconstruction. Knee Surgery, Sports Traumatology, Arthroscopy, 25, 2004-2012. https://doi.org/10.1007/s00167-017-4539-1 